\title{
Asociación de la riqueza y diversidad de especies de aves y estructura de la vegetación en una selva mediana subperennifolia en el centro de Veracruz, México
}

\section{Association of the richness and diversity of bird species and vegetation structure in semiervergreen forest from central Veracruz, Mexico}

\author{
José Cruz Bojorges-Baños ${ }^{1 *}$ y Lauro López-Mata² \\ ${ }^{1}$ Instituto de Recursos, Universidad del Mar, Campus Puerto Escondido, Km. 2.5, carretera a Sola de Vega, San Pedro Mixtepec 71980, Oaxaca, \\ México. \\ 2Programa de Botánica, Colegio de Postgraduados. Montecillo 56230, Estado de México, México. \\ *Correspondencia: jbanos@colpos.mx, josebojorges@zicatela.umar.mx
}

\begin{abstract}
Resumen. Analizamos el grado de asociación de la riqueza y diversidad de especies de aves y la estructura de la vegetación (riqueza y diversidad de especies y de altura); estos atributos se cuantificaron con 12 puntos de conteo (4/área) y con punto centrado en cuadrante (40/área) respectivamente en 3 áreas con diferentes grado de desarrollo. Se estimó la diversidad de especies (Shannon-Wiener) y se compararon estadísticamente la riqueza, abundancia y diversidad de ambas comunidades; la prueba de la relación entre conjuntos de variables se evaluó con análisis de correlación. La inexistencia de correlación entre los atributos avifaunísticos y la diversidad de altura de la vegetación y la evidencia cuantitativa entre la riqueza y diversidad de especies de vegetación y de aves, sugieren que sólo la composición florística es importante en la estructura de la comunidad de aves en este ambiente y a esta escala de análisis. Sugerimos el mantenimiento de parches de vegetación con distintas fases de regeneración natural ya que éstos serían ocupados tanto por diferentes gremios alimentarios como por especies con hábitats específicos y podrían ser necesarios para la supervivencia y reproducción de muchas especies de aves en Santa Gertrudis.
\end{abstract}

Palabras clave: hábitat, aves de Veracruz, correlación.

\begin{abstract}
We analyzed the degree of association of the birds species richness and diversity and the vegetation structure (richness and diversity of species and height); these attributes were quantified respectively with 12 points of count (4/area) and with point centred quarter method (40/area) in 3 areas with different degrees of succession. The species diversity was estimated (Shannon-Wiener) and the richness, abundance and diversity of both communities were statistically compared; the test of the relation between sets of variables was evaluated with correlation analysis. There was no correlation between the bird community and the vegetation height diversity but the quantitative evidence show relation between the richness and species diversity of vegetation and bird community. This suggests only floristic composition is important for the structure of the bird community in this habitat and on this scale of analysis. We suggested the conservation of patches of vegetation with different phases of natural regeneration since these would be taken by different feeding guilds as well as by species with specific habitats and could be necessary for the survival and reproduction of many birds species in Santa Gertrudis.
\end{abstract}

Key words: habitat, birds of Veracruz, correlation.

\section{Introducción}

La variación de la riqueza y diversidad de especies de aves ha sido correlacionada con el clima y la heterogeneidad del paisaje y se ha sugerido que tales factores influyen

Recibido: 13 agosto 2004; aceptado: 05 julio 2006 con diferente magnitud en relación a su escala de análisis (Böhning-Gaese, 1997; Cueto y de Casenave, 1999; Gillespie y Walter, 2001). En este sentido, el clima parece tener gran importancia a nivel macrogeográfico, mientras que regionalmente la heterogeneidad del paisaje tiene mayor relevancia (Böhning-Gaese, 1997; Cueto y de Casenave, 1999). Por otra parte, en estudios 


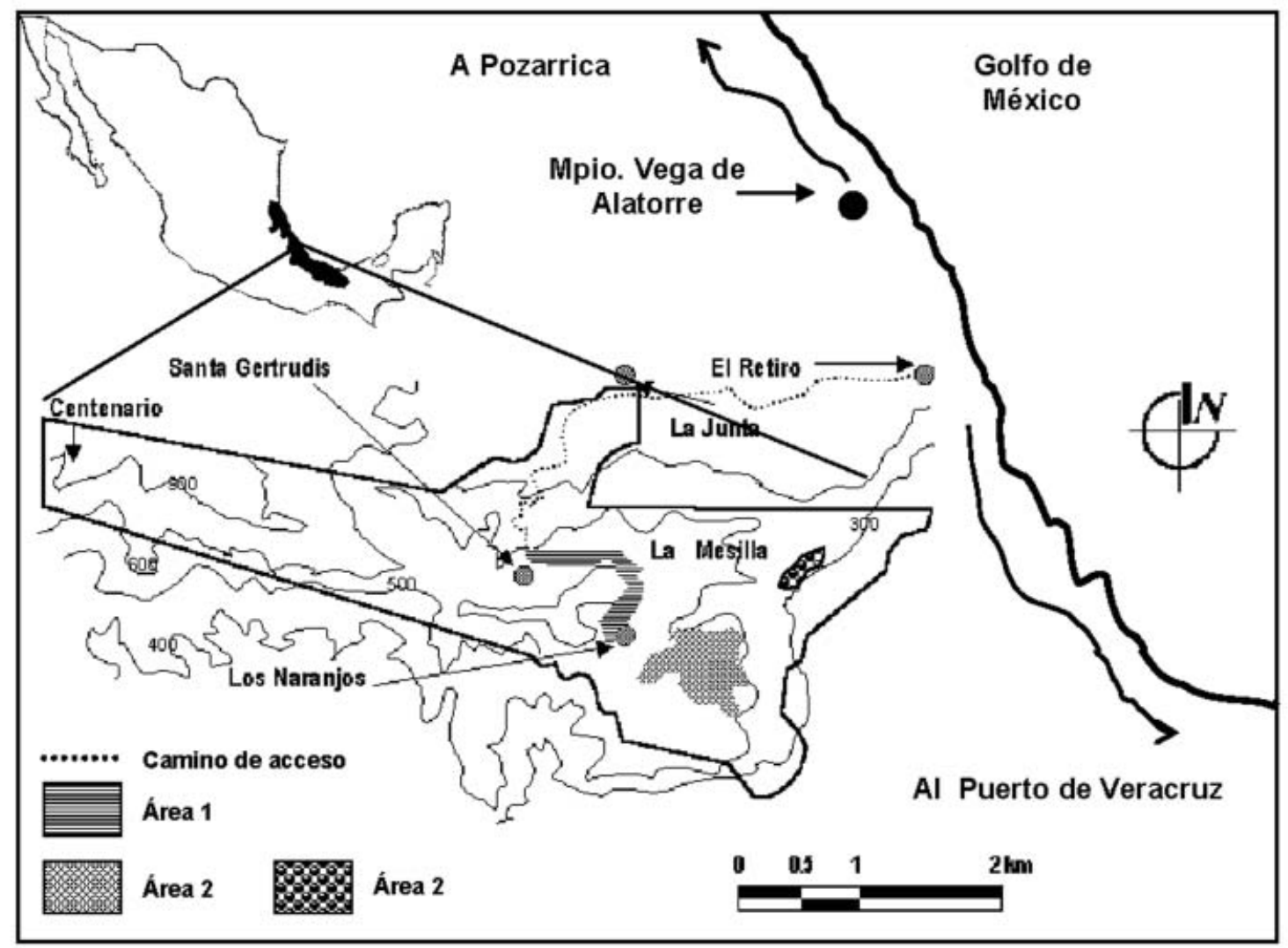

Figura 1. Localización de la zona. Áreas de estudio: 1 parches de potrero, 2, porción de selva con desarrollo intermedio, 3 selva madura.

realizados dentro del hábitat, la riqueza y la diversidad de especies vegetales y de la altura de la vegetación, han sido identificados como buenos indicadores de la riqueza de especies de aves (MacArthur y MacArthur, 1961; MacArthur, 1964; Karr, 1968; Recher, 1969; Karr y Roth, 1971; Roth, 1976; Rotenberry, 1985; Bersier y Meyer, 1994, 1995; Rodríguez-Estrella et al., 1996, García et al., 1998).

Aún cuando existe evidencia de lo anterior, en México son pocos los trabajos que correlacionan la riqueza y diversidad de especies de aves con aspectos estructurales de la vegetación (García et al., 1998; Smith et al., 2001; Moya, 2002) y no existen trabajos de esta índole en selvas medianas subperennifolias. Los trabajos realizados en este tipo de vegetación documentan la riqueza de especies de aves terrestres residentes y migratorias (Lynch 1989, López-Ornat, 1990, Ortiz-Pulido et al., 1995, Bojorges y López-Mata, 2001), lo que ha permitido reconocer su importancia como ambientes diversos hasta con 396 especies en Veracruz; esta riqueza es superada sólo por la riqueza de especies de aves registradas en selvas altas y bosques mesófilos en este estado (Alcántara, 1993). No obstante, no existe información suficiente de estos ambientes que permita sugerir si los cambios de la riqueza y diversidad de especies de aves, están vinculados a los cambios estructurales de la vegetación sujeta a disturbios recurrentes, tanto naturales como antropogénicos (Schulte y Niemi, 1998; Chettri et al., 2002).

Debido a que la avifauna es afectada por la alteración antropogénica de las selvas, comprender la estructura de las comunidades de aves y sus relaciones con la vegetación puede ayudar a identificar tanto patrones de riqueza de especies de aves, como elementos que permitan sugerir estrategias con implicaciones significativas para su conservación (Gillespie y Walter, 2001).

Para sugerir que la riqueza y diversidad de especies de aves presenta un patrón discernible y asociado con los patrones de disturbio de la vegetación y establecer si la diversidad de especies de vegetación corresponde a la diversidad de especies de aves, el presente estudio caracterizó elementos del hábitat asociados a la riqueza y diversidad de especies de aves a escala local, y analizó la relación de la comunidad de aves (riqueza, abundancia y diversidad de especies) y la estructura de la vegetación (riqueza y diversidad de especies y de altura) en tres áreas que representan fases de regeneración con diferente 
desarrollo, ubicadas en los últimos remanentes de selva mediana subperennifolia en el límite latitudinal norte de las selvas tropicales húmedas de México.

\section{Materiales y métodos}

La zona de estudio conocida localmente como Santa Gertrudis tiene una superficie de 930 ha y se ubica entre los $19^{\circ} 49^{\prime}-19^{\circ} 51^{\prime} \mathrm{N}$ y los $96^{\circ} 32^{\prime}-96^{\circ} 37^{\prime} \mathrm{O}$, a una altitud de 400 a 900 m (Fig. 1). El clima es semicálido con temperatura media anual de $22^{\circ} \mathrm{C}$. La precipitación anual es de $2275 \mathrm{~mm}$ y alrededor del $68 \%$ se presenta de junio a noviembre (García, 1988). La vegetación dominante es selva mediana subperennifolia (Miranda y Hernández X., 1963), y de diciembre a abril del 25 al 50\% de los árboles pierden su follaje (Pennington y Sarukhán, 1998).

La riqueza y diversidad de especies de aves se determinó en ambientes diferentes (Remsen, 1994) seleccionados con base en el grado de perturbación y análisis visual de la estructura de la vegetación, asegurándose que tuvieran proporciones continuas. Éstos representan 3 áreas con diferente desarrollo: potrero, selva con regeneración intermedia y selva madura.

El potrero tiene una superficie aproximada de 70 ha, fue creado a principios de 1960 para actividades ganaderas y periódicamente se somete a labores de quema. La vegetación es abierta, de fácil acceso y en su mayoría tiene alrededor de $1 \mathrm{~m}$ de altura, aunque pueden encontrarse arbustos hasta de 5 metros. Son comunes los individuos de los géneros Acacia (Fabaceae), Aechmea (Bromeliaceae), Capsicum (Solanaceae), Lantana (Verbenaceae), Senecio (Asteraceae) y Stenocereus (Cactaceae). Excepcionalmente pueden desarrollarse ahí individuos de Guazuma ulmifolia (Sterculiaceae), Symplocos coccinea (Symplocaceae) y Tabernaemontana alba (Apocynaceae).

El área de selva con regeneración intermedia probablemente está influenciada por la incidencia diferencial de fuertes vientos (Godínez-Ibarra y LópezMata, 2002). El sotobosque lo caracterizan Litsea glaucescens (Lauraceae), Nectandra ambigens (Lauraceae) y Picramnia andicola (Simaroubaceae); este estrato alberga al $90 \%$ de los individuos con alturas menores o iguales a $10 \mathrm{~m}$, por lo que la visibilidad se obstruye a más de $10 \mathrm{~m}$ de distancia. El dosel intermedio lo constituyen individuos de Aphananthe monoica (Ulmaceae), Bursera simaruba (Burseraceae), Croton soliman (Euphorbiaceae) y Dendropanax arboreus (Araliaceae), de 10-20 m de altura. El dosel superior está constituido por individuos con alturas mayores a los $20 \mathrm{~m}$ y lo caracterizan $A$. monoica, B. simaruba, Cecropia obtusifolia (Moraceae), Cedrela odorata (Meliaceae), D. arboreus, y Ficus glabra (Moraceae) (Godínez-Ibarra y López-Mata, 2002). Esta condición se presenta en la mayor parte de la zona de estudio.

El área de selva madura es un parche de alrededor de 13 ha y los elementos arbóreos sobrepasan los $30 \mathrm{~m}$ de altura. La mayoría de los individuos se localizan en el dosel intermedio y superior, permitiendo una visibilidad de hasta $35 \mathrm{~m}$ de distancia o más. El dosel es cerrado y la entrada de luz al sotobosque es escasa. El sotobosque lo integran individuos de Aechmea magdalenae (Bromeliaceae), Brosimum alicastrum (Moraceae), Chamaedorea tepejilote (Arecaceae) y Manilkara zapota (Sapotaceae) con alturas iguales o menores a 10 metros. El dosel intermedio lo componen individuos de A. monoica y Pimenta dioica (Myrtaceae) con alturas entre 10 y 20 metros. El dosel superior está constituido por individuos de B. alicastrum y M. zapota con alturas mayores a los 20 metros (Marín, 2002).

El trabajo de campo comprendió 78 días; de noviembre de 1999 a abril de 2000 se muestreó una semana por mes, y de octubre de 2001 a diciembre de 2002, una semana cada 2 meses; se omitieron julio, agosto y septiembre debido a la inaccesibilidad de la zona en esa temporada de lluvia. La frecuencia y duración de los muestreos se determinaron con base en la previa documentación de las especies (Bojorges y López-Mata, 2001) y con criterios modificados de Gómez de Silva y Medellín (2001).

Muestreo de avifauna. En cada área se ubicaron 4 sitios de muestreo con superficie de 1 ha y separación mínima de $200 \mathrm{~m}$ entre sí. En cada sitio se estableció un punto de conteo (de 1 ha) para el registro y recuento de aves y se colocaron 4 redes de niebla para la captura de individuos que no pudieran ser detectadas visualmente; la combinación de ambos métodos permite registrar un mayor número de especies (Whitman et al., 1997). Estas actividades se realizaron en cada sitio de muestreo durante 6 horas a partir del amanecer, y de las 16:00 horas hasta el anochecer. Se dedicó media hora a cada actividad y 2 días a cada área.

De manera visual, las especies de aves se determinaron mediante su cotejo con las guías de campo de Howell y Webb (1995) y National Geographic (1999), y de manera auditiva, con los cantos de las mismas especies en grabaciones de Coffey y Coffey (1989). La riqueza de especies de aves para cada área se obtuvo sumando las especies observadas y capturadas dentro de los sitios de muestreo (Bojorges y López-Mata, 2005); la estacionalidad se determinó como residente y migratoria (Howell y Webb, 1995) y para la nomenclatura se empleó la de la Unión de Ornitólogos Americanos (AOU, 2002).

Para tener idea de la abundancia relativa de la avifauna observada se tomaron en cuenta únicamente los datos de los 
recuentos (excluyendo las aves capturadas) y se agruparon en las siguientes categorías: especies abundantes, las registradas siempre con 15 individuos o más; escasas, las no siempre registradas con 5-14 individuos, y raras, las registradas con 4 individuos o menos (Coates-Estrada et al., 1985).

Para cada área y sitio de muestreo se realizó lo siguiente: 1) se estimó la riqueza de especies con el Indicador de Cobertura basado en Incidencia (ICI) en el programa EstimateS (Colwell, 2000) con datos de riqueza y abundancia; el estimador se basa en el concepto estadístico de cobertura de muestra e infiere la riqueza de especies con relativamente pocas unidades de muestreo (Chazdon et al., 1998); 2) se determinó la similitud de la riqueza con el Coeficiente de Comunidad de Jaccard por su confiabilidad en el análisis de datos de presencia ausencia (MuellerDombois y Ellenberg, 1974); 3)se detectaron diferencias significativas entre la abundancia de especies con análisis de Kruskal-Wallis (SAS, 1999), y 4) con los datos de riqueza y la abundancia de cada especie obtenida en los recuentos, se estimó el índice de diversidad de ShannonWiener (H'). Los valores de diversidad $\left(\mathrm{H}^{\prime}\right)$ obtenidos fueron comparados con pruebas de t de Hutcheson (Zar, 1996).

Muestreo de la vegetación. Se utilizó el método de punto centrado en cuadrante (Muller-Dombis y Ellenberg, 1974; Bersier y Meyer, 1994), estableciendo 10 de éstos en cada sitio de muestreo $(n=40$ por área). Las especies fueron identificadas in situ por su nombre común y se determinaron tanto la abundancia como la altura de cada individuo; con estos datos se determinó la riqueza y se estimaron índices de diversidad (Shannon-Wiener) tanto de especies como de altura de la vegetación (diversidad de altura de vegetación) en cada área y sitio de muestreo. Los valores de diversidad (H') obtenidos en cada área y sitio fueron comparados con pruebas de t de Hutcheson (Zar, 1996). La similitud de la riqueza de especies se determinó con el Coeficiente de Comunidad de Jaccard. Los valores de diversidad, tanto de especies de aves como de la vegetación, así como de la altura de la misma, se refieren al índice de diversidad de Shannon-Wiener.

Para determinar el grado de asociación entre las variables avifáunicas y las variables de la estructura de la vegetación se realizaron análisis de correlación $(\mathrm{N}=12)$, considerando para ello las variables cuantificadas (riqueza, abundancia y diversidad de especies de aves y de vegetación y diversidad de altura de vegetación) en cada sitio de muestreo, previo examen de la normalidad en la distribución de los datos (Naranjo y Chacón de Ulloa, 1997).

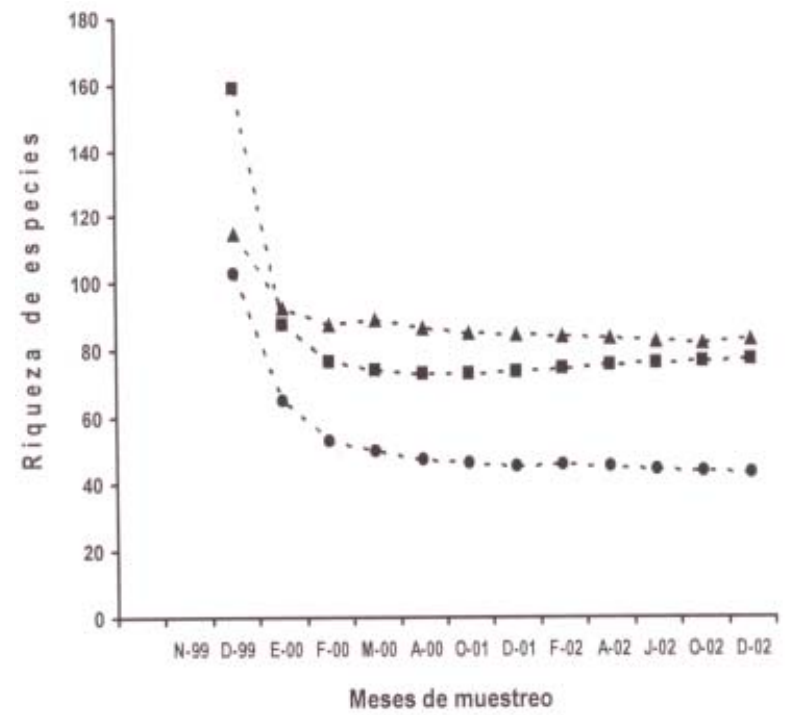

Figura 2. Riqueza de especies estimada en cada área de muestreo. Potrero (cuadrado), selva en regeneración (triángulo), selva madura (circunferencia).

\section{Resultados}

Avifauna. Durante 660 horas de observación se registró un total de 2512 individuos de 114 especies pertenecientes a 30 familias de 13 órdenes. De estas especies, 70 (61\%) fueron residentes y 44 (39\%) migratorias; 68 (60\%) fueron abundantes, $24(21 \%)$ escasas y $22(19 \%)$ raras. Las familias con mayor número de especies fueron Parulidae con 22, Tyrannidae con 11, y Vireonidae con 7 (Apéndice). El número total de horas red fue de 2340; se capturaron 551 individuos en las 3 áreas (320 en la 2, 193 en la 1 y 38 en la 3) pertenecientes a 46 especies; a excepción de Catharus mexicanus, C. minimus y Geotrygon montana, las cuales sólo se registraron por este medio en la selva con regeneración intermedia, las demás especies capturadas en cada una de las áreas también fueron vistas y/o escuchadas.

El estimador de riqueza de especies sugiere que en cada área se registró la mayoría de las especies que ahí podrían encontrarse; en la selva con regeneración intermedia se registraron casi todas, mientras que en el potrero y en la selva madura faltarían por registrar alrededor de 8 y 2 especies, respectivamente (Fig. 2).

En el potrero se observaron 606 individuos de 70 especies (41 residentes y 29 migratorias) y una diversidad de 3.86. Las especies más abundantes en esta área fueron Cardinalis cardinalis, Crotophaga sulcirostris, entre otras (Apéndice 1). La riqueza más alta se registró en el sitio 4 (42 especies) y tanto la abundancia como la 
Cuadro 1. Riqueza, abundancia y diversidad (H') de especies de aves y de vegetación registradas por área y sitio de estudio. Áreas: $1=$ potrero, $2=$ selva con regeneración intermedia, $3=$ selva madura

\begin{tabular}{|c|c|c|c|c|c|c|c|}
\hline \multirow[b]{2}{*}{ Áreas } & \multirow[b]{2}{*}{ Sitios } & \multicolumn{3}{|c|}{ Avifauna } & \multicolumn{3}{|c|}{ Vegetación } \\
\hline & & Riqueza & Abundancia & Diversidad & Riqueza & Diversidad & Diversidad-altura \\
\hline \multirow[t]{4}{*}{1} & 1 & 37 & 151 & $3.46^{\mathrm{a}}$ & 17 & $2.57^{\mathrm{a}}$ & $2.20^{\mathrm{a}}$ \\
\hline & 2 & 30 & 128 & $3.26^{\mathrm{b}}$ & 12 & $1.94^{\mathrm{b}}$ & $2.11^{\mathrm{a}}$ \\
\hline & 3 & 40 & 167 & $3.54^{\mathrm{ac}}$ & 17 & $2.53^{\mathrm{a}}$ & $2.16^{\mathrm{a}}$ \\
\hline & 4 & 42 & 162 & $3.52^{\mathrm{ac}}$ & 9 & $1.34^{\mathrm{c}}$ & $1.78^{\mathrm{b}}$ \\
\hline Por área & & 70 & 606 & 3.86 & 32 & 2.85 & 2.34 \\
\hline \multirow[t]{4}{*}{2} & 1 & 59 & 475 & $3.75^{\mathrm{a}}$ & 20 & $2.64^{\mathrm{a}}$ & $3.0^{\mathrm{a}}$ \\
\hline & 2 & 55 & 422 & $3.66^{\mathrm{ac}}$ & 20 & $2.70^{\mathrm{ac}}$ & $2.64^{\mathrm{be}}$ \\
\hline & 3 & 41 & 305 & $3.49^{b}$ & 17 & $2.53^{\mathrm{a}}$ & $2.64^{\mathrm{ce}}$ \\
\hline & 4 & 54 & 449 & $3.62^{\mathrm{c}}$ & 13 & $2.18^{\mathrm{b}}$ & $2.70^{\mathrm{de}}$ \\
\hline Por área & & 81 & 1651 & 3.89 & 41 & 3.30 & 3.37 \\
\hline \multirow[t]{4}{*}{3} & 1 & 27 & 76 & $3.10^{\mathrm{a}}$ & 9 & $1.77^{\mathrm{a}}$ & $2.88^{\mathrm{a}}$ \\
\hline & 2 & 26 & 67 & $2.99^{a b c}$ & 14 & $2.35^{\mathrm{b}}$ & $2.70^{\mathrm{a}}$ \\
\hline & 3 & 22 & 61 & $2.74^{\mathrm{bc}}$ & 9 & $1.90^{\mathrm{a}}$ & $2.55^{b c}$ \\
\hline & 4 & 19 & 47 & $2.58^{\mathrm{c}}$ & 7 & $1.35^{\mathrm{c}}$ & $2.68^{\mathrm{ac}}$ \\
\hline Por área & & 40 & 253 & 3.37 & 20 & 2.18 & 3.23 \\
\hline
\end{tabular}

Cuadro 2. Similitud de la riqueza de especies entre sitios de muestreo. Áreas: $1=$ potrero, $2=$ selva con regeneración intermedia, $3=$ selva madura

\begin{tabular}{|c|c|c|c|c|c|c|c|c|c|}
\hline & \multicolumn{3}{|c|}{1} & \multicolumn{3}{|c|}{2} & \multicolumn{3}{|c|}{3} \\
\hline & Sitios & $\begin{array}{l}\text { Especies } \\
\text { comunes }\end{array}$ & Similitud & Sitios & $\begin{array}{l}\text { Especies } \\
\text { comunes }\end{array}$ & Similitud & Sitios & $\begin{array}{l}\text { Especies } \\
\text { comunes }\end{array}$ & Similitud \\
\hline \multirow[t]{6}{*}{ Avifauna } & 1 y 2 & 23 & 0.52 & 1 y 2 & 42 & 0.58 & 1 y 2 & 18 & 0.51 \\
\hline & 1 у 3 & 24 & 0.45 & 1 y 3 & 36 & 0.56 & 1 y 3 & 17 & 0.56 \\
\hline & 1 y 4 & 25 & 0.46 & 1 y 4 & 40 & 0.54 & 1 y 4 & 12 & 0.37 \\
\hline & 2 y 3 & 17 & 0.32 & 2 y 3 & 35 & 0.57 & 2 y 3 & 14 & 0.43 \\
\hline & 2 y 4 & 18 & 0.33 & 2 y 4 & 41 & 0.60 & 2 y 4 & 11 & 0.34 \\
\hline & 3 y 4 & 25 & 0.43 & 3 y 4 & 35 & 0.58 & 3 y 4 & 12 & 0.48 \\
\hline \multirow[t]{6}{*}{ Vegetación } & 1 y 2 & 7 & 0.31 & 1 y 2 & 9 & 0.29 & 1 y 2 & 7 & 0.43 \\
\hline & 1 y 3 & 8 & 0.30 & 1 y 3 & 7 & 0.23 & 1 y 3 & 6 & 0.46 \\
\hline & 1 y 4 & 4 & 0.18 & 1 y 4 & 4 & 0.13 & 1 y 4 & 3 & 0.25 \\
\hline & 2 y 3 & 6 & 0.26 & 2 у 3 & 9 & 0.32 & 2 y 3 & 6 & 0.33 \\
\hline & 2 y 4 & 3 & 0.16 & 2 y 4 & 5 & 0.17 & 2 y 4 & 3 & 0.17 \\
\hline & 3 y 4 & 5 & 0.22 & 3 y 4 & 6 & 0.25 & 3 y 4 & 5 & 0.45 \\
\hline
\end{tabular}


diversidad más altas se registraron en el sitio 3 con 167 y 3.54 respectivamente. La diversidad del sitio 2 fue la más baja de esta área y presentó diferencias $(\mathrm{p}<0.05)$ con los demás sitios (Cuadro 1). La abundancia de especies no presentó diferencias entre los sitios de muestreo ( $\mathrm{p}>0.05)$. La similitud entre los sitios 1 y 2 fue la más alta con 0.52 y compartieron 23 especies (Cuadro 2).

En la selva con regeneración intermedia se registraron la mayor riqueza, abundancia y diversidad con 81 (54 residentes y 27 migratorias), 1651 y 3.89 respectivamente. Las especies más abundantes en está área fueron Cyanocompsa parellina, Psarocolius montezuma entre otras (Apéndice). Asimismo, en el sitio 1 se observaron la mayor riqueza (59), abundancia (475) y diversidad de especies (3.75). La diversidad del sitio 3 fue la más baja y difirió $(\mathrm{p}<0.05)$ de los otros sitios (Cuadro 1). La abundancia de especies entre los sitios de muestreo no presentó diferencias ( $\mathrm{p}>0.05)$. La similitud entre los sitios 2 y 4 fue la más alta con 0.60 y compartieron 41 especies (Cuadro 2).

En la selva madura se registraron la menor riqueza, abundancia y diversidad de especies con 40 (28 residentes y 12 migratorias), 253 y 3.37 respectivamente. Las especies más abundantes en esta área fueron Basileuterus culicivorus, Euthlypis lacrimosa, entre otras (Apéndice). La riqueza, la abundancia y la diversidad más altas se obtuvieron en el sitio 1 , con 27,76 y 3.10 respectivamente. La diversidad estimada en el sitio 3 fue la más baja y mostró diferencias $(\mathrm{p}<0.05)$ con otros sitios (Cuadro 1). La abundancia de especies entre los sitios de muestreo no presentó diferencias ( $\mathrm{p}>0.05)$. La similitud entre los sitios 1 y 3 fue la más alta con 0.56 y compartieron 17 especies (Cuadro 2).

Al comparar las 3 áreas, se observa que la diversidad de especies estimada en la selva madura difiere tanto de la diversidad del potrero $\left(\mathrm{t}_{418(2)}=8.26, \mathrm{p}<0.05\right)$ como de la selva con regeneración intermedia $\left(\mathrm{t}_{353(2)}=8.88\right.$, $\mathrm{p}<0.05)$. Sin embargo, estas últimas fueron similares $\left(\mathrm{t}_{1383(2)}=0.29, \mathrm{p}>0.05\right)$, mientras que la abundancia de las 3 áreas fue diferente $(\mathrm{p}<0.05)$. El apéndice muestra las especies registradas en cada área y sitio. El área de potrero comparte con el área de selva en regeneración intermedia 42 especies y son $37 \%$ similares, mientras que con la selva madura comparte 22 especies con $25 \%$ de similitud. Por otra parte, las áreas de selva en regeneración intermedia y madura compartieron 35 especies y fueron $40 \%$ similares.

Vegetación. Se registraron 67 especies en las 3 áreas. En el potrero, la riqueza y diversidad de especies y de altura de vegetación fue de 32, 2.85 y 2.34 respectivamente; esta última fue la más baja de las 3 áreas. La riqueza y diversidad de especies y de altura de vegetación más altas en esta área se registraron en el sitio 1 (Cuadro 1). La diversidad de especies y de altura de vegetación más bajas se estimaron en el sitio 4 y difirieron $(\mathrm{p}<0.05)$ de los otros sitios (Cuadro 1). La similitud entre los sitios 1 y 2 fue la más alta, con 0.31, al compartir 7 especies (Cuadro 2).

En el área de regeneración intermedia se obtuvo la riqueza y diversidad de especies y de altura de la vegetación más altas con 41, 3.30 y 3.37 respectivamente; la mayor riqueza y diversidad de especies se registraron en el sitio 2 , mientras que la diversidad de altura de la vegetación más alta se observó en el 1 (Cuadro 1). La diversidad de especies del sitio 4 fue la más baja y difirió significativamente de los demás sitios; asimismo, la diversidad de altura de

Cuadro 3. Coeficientes de correlación de Pearson entre las variables avifáunicas y de vegetación.

\begin{tabular}{llll}
\hline Vegetación & Avifauna & & \\
\hline & Riqueza & Abund. & Diversidad \\
Riqueza & $0.77903 *$ & $0.73672 *$ & $0.77175^{*}$ \\
Diversidad & $0.64762 *$ & $0.64630 *$ & $0.62615^{*}$ \\
Diversidad altura & 0.04150 & 0.22646 & -0.13528 \\
\hline
\end{tabular}

$* P<0.05$

vegetación de los sitios 2 y 3 fueron la más bajas y difirieron $(\mathrm{p}<0.05)$ de los demás sitios (Cuadro 1). La similitud entre los sitios 2 y 3 fue la más alta con 0.32 y compartieron 9 especies (Cuadro 2).

En el área de selva madura se registraron la menor riqueza y diversidad de especies con 20 y 2.18 respectivamente, mientras que la diversidad de altura de la vegetación fue de 3.32 .

La riqueza y diversidad de especies y de altura de vegetación tuvieron sus valores más altos en los sitios 2 y 1 respectivamente (Cuadro 1). La diversidad de especies y de altura de vegetación tuvieron sus valores más bajos en los sitios 4 y 3 respectivamente, y en ambos casos difieren estadísticamente $(\mathrm{p}<0.05)$ de los demás sitios (Cuadro 1$)$. La similitud entre los sitios 1 y 3 fue la más alta con 0.46 y compartieron 3 especies (Cuadro 2).

Al comparar las 3 áreas se observó que la diversidad de especies estimada en el potrero difiere del área de selva en regeneración intermedia $\left(\mathrm{t}_{205(2)}=2.13, \mathrm{p}<0.05\right)$ y del área de selva madura $\left(\mathrm{t}_{230(2)}=3.08, \mathrm{p}<0.05\right)$; asimismo, estas últimas mostraron diferencias $\left(\mathrm{t}_{21(2)}=9.27, \mathrm{p}<0.05\right)$. La diversidad de altura de la vegetación en el potrero difirió de las áreas con regeneración intermedia $\left(\mathrm{t}_{655(2)}=\right.$ 
9.32, $\mathrm{p}<0.05)$ y selva madura $\left(\mathrm{t}_{320(2)}=11.05, \mathrm{p}<0.05\right)$; por el contrario, estas últimas no presentaron diferencias $\left(\mathrm{t}_{684(2)}=1.56, \mathrm{p}>0.05\right)$. Adicionalmente, el área de potrero compartió 12 especies y fue $19 \%$ similar con la selva en regeneración intermedia, mientras que con la selva madura compartió 3 especies y fueron $6 \%$ similares. Por otra parte, las áreas de selva en regeneración intermedia y madura compartieron 14 especies y fueron $29 \%$ similares.

La correlación mostró la existencia de asociación significativa entre la riqueza y diversidad de especies de vegetación y la riqueza, diversidad y abundancia de especies de aves; por el contrario, la diversidad de altura de la vegetación no estuvo asociada a las variables avifáunicas (Cuadro 3).

\section{Discusión}

La combinación de registro visual y auditivo utilizado en este estudio está considerado como un método eficiente para realizar inventarios en bosques tropicales (Stiles y Bohórquez, 2000). El uso de grabaciones para identificar especies y las capturas agregaron pocas especies no registradas por los otros métodos. Lo anterior permite argumentar que no existen sesgos severos en el registro de la riqueza de especies en cada sitio. La convergencia cercana de las curvas de acumulación de especies, tanto observadas como estimadas, apoyan el argumento anterior. Se observó un decremento de especies únicas y duplicadas en el transcurso de los muestreos, lo que indica, de acuerdo con el estimador, que la riqueza está mejor representada conforme las especies raras son menos frecuentes, (Longino et al., 2002).

La relativamente baja similitud entre las áreas de estudio sugiere que cada una presenta su propia composición de especies de aves; sin embargo, la variación de la riqueza y sus abundancias son atribuibles al intercambio de especies (Blake y Loiselle, 2001) que influye en la composición de la comunidad de aves y a las diferencias locales, tales como la heterogeneidad del hábitat (Böhning-Gaese, 1997). En este sentido, el mosaico de vegetación permite a las aves cambiar su distribución en respuesta a las condiciones del ambiente (Karr, 1990), utilizándolo para diferentes propósitos (Staicer, 1992). La relativa cercanía de las áreas de potrero y de selva con regeneración intermedia apoya este argumento, ya que éstas comparten la mayor cantidad de especies. Por otra parte, el $85 \%$ de las especies registradas en la selva madura también se observaron en la selva con regeneración intermedia, lo que sugiere que no tienen preferencia de hábitat específico, como aquellas que se registraron solamente en una área. Lo anterior hace evidente una conectividad entre estas áreas (sensu
Wiens, 1997) y sugiere que la configuración del hábitat (e.g. paisaje, hábitat y microhábitat) (Karr, 1990 ) es tan importante en la distribución de las aves como la cantidad de ambientes disponibles (Sherry y Holmes, 1995; Bersier y Meyer, 1995).

Los valores más altos de ambos grupos de variables (avifauna y vegetación) registrados en el área de selva con regeneración intermedia pueden ser consecuencia de un disturbio moderado, probablemente influenciado por la incidencia diferencial de fuertes vientos (GodínezIbarra y López-Mata, 2002), el cual puede incrementar la diversidad de especies.

Por otra parte, la equidad estimada en las áreas de potrero y selva con regeneración intermedia fue similar, por lo que no se encontró diferencia estadística entre la diversidad de especies de aves entre estas áreas (Bojorges y López-Mata, 2005). Lo anterior concuerda con lo registrado por Cueto (1996), quien estableció que en ambientes degradados se presenta una equidad mayor que en ambientes conservados. En otro sentido, las diferencias significativas entre la diversidad de especies de aves de la selva madura, respecto al potrero y a la selva con regeneración intermedia, se debieron a que en la primera se registró la menor riqueza y abundancia de especies, lo que puede atribuirse a que esta área es menos extensa que las otras y sugiere que tanto la riqueza como la abundancia de especies se abaten a medida que la superficie decrece, como se ha documentado en diferentes ambientes (Newmark, 1991; Adler, 1994).

Los resultados del presente trabajo indican que tanto la riqueza como la abundancia y diversidad de especies de aves estuvieron asociadas únicamente con la riqueza y diversidad de especies de plantas. Lo anterior contrasta con lo establecido por MacArthur (1964), Karr (1968), Recher (1969), Karr y Roth (1971), Willson (1974), Roth (1976) y Rotenberry (1985), quienes consideraron que a pequeña escala la composición de las comunidades de aves y la abundancia de muchas especies están estrechamente influenciadas por la riqueza y diversidad de especies y de altura de la vegetación. Sin embargo, los hallazgos aquí presentados apoyan los trabajos de Snow y Snow (1971), Bersier y Meyer (1995) y Rodríguez-Estrella et al. (1996), quienes mencionaron que la diversidad de altura de vegetación no se correlacionó con ninguna variable avifaunística. A este respecto, Bersier y Meyer (1994) anotan que este atributo es menos importante en la determinación de la riqueza y diversidad de especies de aves a medida que el hábitat se reduce en superficie.

La diversidad de especies de aves registrada en las áreas de potrero y de selva con regeneración intermedia apoyan lo anterior, este atributo fue similar aún cuando existieron diferencias significativas entre la diversidad 
de altura de vegetación de estas áreas. Adicionalmente, se ha señalado que hábitats con similar diversidad de altura de vegetación presentan similar riqueza de especies de aves (MacArthur y MacArthur, 1961); sin embargo, dicha tendencia no se observó entre las áreas de selva con regeneración intermedia y selva madura.

Por otra parte, contrario a lo establecido por Bell (1979), la relativamente homogénea diversidad de altura de vegetación del área de potrero, en la cual los elementos muestreados no excedieron los $5 \mathrm{~m}$, no influyó en la disminución de la diversidad de especies, ya que, como se mencionó, ésta es similar a la de la selva con regeneración intermedia. En este sentido, se ha concluido que la presencia de ciertas especies de plantas influyen de manera más importante en la estructura de las comunidades de aves que la estructura de la vegetación misma (Rice et al., 1994). A diferencia de potreros desprovistos de árboles, los cuales son pobres en riqueza de especies de aves (Estrada et al., 1997), los potreros con árboles en pie proporcionan sitios de percha y recursos alimenticios (Lynch, 1989). Adicionalmente, se ha documentado que la presencia de árboles de Acacia proporciona un excelente hábitat, el cual es preferido por aves migratorias como Dendroica magnolia, D. virens y Polioptila caerulea (Greenberg et al., 1997).

La inexistencia de correlación entre la riqueza, abundancia y diversidad de aves y la diversidad de altura de la vegetación, así como la evidencia cuantitativa que establece nexos entre la riqueza y diversidad de especies de vegetación y avifauna, sugiere que sólo la composición florística influye de manera importante en la estructura de la comunidad de aves en este ambiente y a esta escala de análisis. Lo anterior apoya la hipótesis de Rotenberry (1985), quien menciona que a pequeña escala, la riqueza y diversidad de especies vegetales influyen en la determinación de la riqueza, abundancia y diversidad de especies de aves. Asimismo, la dinámica de las especies la controla una combinación de factores que las inducen a responder a la variación ambiental. En este sentido, la composición avifaunística observada en este estudio incluye tanto especies únicas o particulares de cada área (e.g. Campylorhynchus rufinucha en potrero, Crax rubra en selva madura) como especies comunes entre áreas (e.g. Henicorhina leucosticta), las cuales dependen de las condiciones estructurales de estas últimas para su establecimiento, alimentación, etc.

La carencia de estudios similares en ambientes semejantes dificulta la comparación de nuestros resultados, por lo que desconocemos si en otras selvas medianas subperennifolias existe un patrón similar. Debido a las variaciones en los métodos de campo y análisis adoptados por los investigadores es casi imposible comparar estudios en los que se correlacionan parámetros de vegetación y aves (Erdelen, 1984). La comparación sólo sería posible si el significado de las variables fuera similar y si las especies de plantas tienen aproximadamente los mismos hábitats (Bersier y Meyer, 1994). Sin embargo, podemos argumentar que en la zona de estudio la riqueza y diversidad de especies de aves registradas en las áreas de selva madura y potrero no presentan un patrón discernible y asociado con los patrones de disturbio de la vegetación. Asimismo, los valores más altos de ambos grupos de variables (avifauna y vegetación) estimados en el área de selva con regeneración intermedia concuerdan con trabajos realizados en condiciones de similares de regeneración y sugieren que la influencia de un disturbio intermedio incrementa la diversidad de especies.

Aún cuando la conservación de aves terrestres depende de la comprensión clara de sus requerimientos de hábitat y de los procesos físicos y bióticos que lo mantienen (Askins, 2000), se ha establecido que la combinación de hábitats simples (potrero) y complejos (áreas de selva) conduce a nuevas posibilidades de explotación diferencial del espacio (Willson, 1974) y que la diversidad de especies de aves está vinculada a la diversidad del paisaje, por lo que la conservación de este último aseguraría la conservación de la diversidad de especies (BöhningGaese, 1997). En este sentido, debido al intercambio de especies que se registró en este estudio y a que cada área aporta especies propias (e. g. Sittasomus griseicapillus en selva madura), puede argumentarse que diferentes ambientes promueven la riqueza y diversidad de especies de aves (Böhninig-Gaese, 1997) al adicionar mayor riqueza y diversidad de especies vegetales (Mills et al., 1991); un bosque denso y "maduro" (sensu Odum), no sería lo ideal para conservar alta diversidad de especies de aves (Chettri et al., 2005) . Por lo anterior, sugerimos que la incorporación y mantenimiento de parches de vegetación con distintas fases de regeneración natural, de formas y tamaños variables, podrían ser necesarios para la supervivencia y reproducción de muchas especies de aves en Santa Gertrudis, ya que éstos serían ocupados tanto por diferentes gremios alimentarios como por especies con hábitats específicos. Adicionalmente, ante la continua fragmentación de hábitats, la prioridad es conservar lo que quede en las condiciones que quede. En este sentido, los esfuerzos de conservación deben enfocarse primero en conservar los fragmentos de selva que se mantienen en el paisaje y al mismo tiempo tratar de aumentar la cobertura arbórea del potrero.

\section{Agradecimientos}

Este proyecto fue financiado por CONACYT bajo 
convenio con L. López-Mata (CP-CONACYT-0443P-N) y por el Instituto de Recursos Naturales del Colegio de Postgraduados. Algunos aspectos de esta investigación fueron apoyados por las Fundaciones Telmex y Hombre y Naturaleza mediante una beca asignada a JCBB. Agradecemos a la MC. Beatriz Aguilar Valdez por el préstamo del equipo de campo, a la familia Hernández Ochoa y al Ing. Bernardo Vega H. por las facilidades brindadas para realizar la investigación en la zona de estudio. Raymundo Espinosa S. y Segundo Zárate C. prestaron su valiosa ayuda durante el trabajo de campo.

\section{Literatura citada}

Alcántara, C. J. L. 1993. Evaluación avifaunística de Veracruz: un análisis de la distribución espacial para la conservación. Tesis de maestría, Facultad de Ciencias, Universidad Nacional Autónoma de México, México, D.F. 201 p.

AOU (American Ornithologists' Union). 2002 Check-list of North American birds. http://www.aou.org/aou/ birdlist.htm

Adler, G. H. 1994. Avifaunal diversity and endemism on tropical Indian Ocean islands. Journal of Biogeography 21:85-95.

Askins, R. A. 2000. Restoring North America's birds. Yale University Press. 336 p.

Bell, H. L. 1979. The effects on rainforest birds of plantings of teak, Tectona grandis, in Papua New Guinea. Australian Wildlife Research 6:305-318.

Bersier, L. F., y D. R. Meyer. 1994. Bird assemblages in mosaic forest: the relative importance of vegetation structure and floristic composition along the successional gradient. Revue d'Ecologie (la Terre et la Vie) 50:15-33.

Bersier, L. F., y D. R. Meyer. 1995. Relation between bird assemblages, vegetation structure and floristic composition of mosaic patches in riparian forest. Acta Oecologica 15:561-576

Bojorges, B. J. C. y López-Mata L. 2001. Abundancia y distribución temporal de aves en una selva mediana subperennifolia en el centro de Veracruz, México. Anales del Instituto de Biología, Universidad Nacional Autónoma de México, Serie Zoología 72:259-283.

Bojorges, B. J. C. y López-Mata L. 2005. Riqueza y diversidad de especies de aves en una selva mediana subperennifolia en el centro de Veracruz, México. Acta Zoológica Mexicana (n.s.) 21:1-20.

Böhning-Gaese, K. 1997. Determinants of avian species richness at different spatial scales. Journal of Biogeography 24:49-60.
Blake, J. G., y B. A. Loiselle. 2001.Bird assemblages in second-growth and old-growth forest, Costa Rica: perspectives from mist nets and point counts. Auk 118:304-326.

Chazdon, R. L., R. K. Colwell, J. S. Denslow y M. Guariguata. 1998. Statistical estimation of species richness of woody regeneration in primary and secondary rainforests of northeastern Costa Rica. In Forest biodiversity in North, Central, and South America and the Caribbean: research and monitoring, F. Dallmeier y J. Comisky (eds.). Parthenon, Paris, p. 285-309.

Chettri, N., D. C. Deb, E. Sharma, y R. Jackson. 2005. The relationship between bird communities and habitat: A study along a trekking corridor of the Sikkim Himalaya. Mountain Research and Development 25:235-244.

Coates-Estrada, R., A. Estrada, D. Pashley y W. Barrow. 1985. Lista de las aves de la Estacion de Biología Los Tuxtlas. Instituto de Biología y Dirección General de Publicaciones. Universidad Nacional Autónoma de México, México, D.F. 41 p.

Coffey, B. B., Jr., y L. C. Coffey. 1989. Songs of Mexican birds. Ara Records, Gainesville, Florida [cassette tape].

Colwell, R. K. 2000. EstimateS: Statistical estimation of species richness and shared species from samples. Version 6. User's guide and application published at: htp://viceroy.eeb.uconn.edu/estimates

Cueto, V. R. 1996. Relación entre los ensambles de aves y la estructura de la vegetación. Un análisis a tres escalas espaciales. Tesis doctorado, Universidad de Buenos Aires. 66 p.

Cueto, V. R. y J. L. de Casenave. 1999. Determinants of bird species richness: role of climate and vegetation structure at a regional scale. Journal of Biogeography 26:487-492.

Erdelen, M. 1984. Bird communities and vegetation structure: I. Correlations and comparisons of simple and diversity indices. Oecologia 61:277-284.

Estrada, A., R. Coates-Estrada y D. A. Meritt Jr. 1997. Anthropogenic landscape changes and avian diversity at Los Tuxtlas, Mexico. Biodiversity and Conservation 6:19-43.

García, E. 1988. Modificaciones al sistema de clasificación climática de Köppen (para adaptarlo a las condiciones de la República Mexicana) Offset Larios, México, D.F. 222 p.

García, S., D. M. Finch, y L. G. Chávez. 1998. Patterns of forest use and endemism in resident bird communities of north-central Michoacan, Mexico. Forest Ecology and Management 110:151-171.

Gillespie, T. W. y H. Walter. 2001. Distribution of bird 
species richness at a regional scale in tropical dry forest of Central America. Journal of Biogeography 28:651-662.

Godínez-Ibarra, O. y L. López-Mata. 2002. Estructura, composición, riqueza y diversidad de especies de árboles en tres muestras de una selva mediana subperennifolia. Anales del Instituto de Biología, Universidad Nacional Autónoma de México, Serie Botánica 73:259-283.

Gómez de Silva, G. H. y Medellín, R. 2001. Evaluating completeness of species list for conservation and macroecology: case-study of Mexican land birds. Conservation Biology 15:1384-1395.

Greenberg, R., P. Bichier y J. Sterling. 1997. Acacia, cattle, and migratory birds in southeastern Mexico. Biological Conservation 80:235-247

Howell, S. N. G. y S. Webb. 1995. A guide to the birds of Mexico and Northern Central America. Oxford University Press, New York. 851 p.

Karr, J. R. 1968. Habitat and avian diversity on strip-mined land in east-central Illinois. Condor 70:348-357.

Karr, J. R. 1990. Birds of tropical rainforest: comparative biogeography and ecology. In Biogeography and ecology of forest bird communities, A. Keast (ed.). SPB Academic, The Hague, p. 215-228.

Karr, J. R. y R. R. Roth. 1971 Vegetation structure and avian diversity in several new world areas. American Naturalist 105:423-435.

Longino, J. T., J. Coddington y R. K. Colwell. 2002. The ant fauna of a tropical rain forest: estimating species richness three different ways. Ecology 83:689-702.

López-Ornat, A. 1990. Avifauna de la Reserva de la Biosfera de Sian Ka'an. In Diversidad biológica en la Reserva de la Biosfera de Sian Ka'an Quintana Roo, México. D. Navarro y J. G. Robinson (eds.). Centro de Investigaciones de Quintana Roo, México, p. 332370.

Lynch, J. F. 1989. Distribution of overwintering neartic migrants in the Yucatan Peninsula, II: use of relative and human-modified vegetation. In Ecology and conservation of neotropical migrant landbirds, J. M.Hagan y D. W. Johnston (eds.). Smithsonian Institution Press, Washington, D. C., p. 178-196.

MacArthur, R. H. 1964. Environmental factors affecting bird species diversity. American Naturalist 98:387397.

MacArthur, R. H. y J. W. Mc Arthur. 1961. On bird species diversity. Ecology 42:594-598.

Marín, C. V. H. 2002. Importancia económica de productos forestales no maderables en una selva mediana subperennifolia en el centro de Veracruz. Departamento de Agroecología. Universidad Autónoma Chapingo,
Estado de México. 106 p.

Mills, G. S., J. B. Dunning y J. M. Bates. 1991. The relationship between breeding bird density and vegetation volume. Wilson Bulletin 103:468-479.

Miranda, F. y E. Hernández, X. 1963. Los tipos de vegetación en México y su clasificación. Boletín de la Sociedad Botánica de México 28:29-179.

Moya, M. H. 2002. Disponibilidad de alimento y estructura del hábitat en la distribución y abundancia de aves insectívoras en una selva baja en Estipac, Jalisco. Tesis maestría, Facultad de Ciencias, Universidad Nacional Autónoma de México, México D.F. 96 p.

Muller-Dombois, D. y H. Ellenberger. 1974. Aims and methods of vegetation ecology. Wiley, New York. 574 p.

Naranjo, L. G. y Chacón de Ulloa. 1997. Diversidad de insectos y aves insectívoras de sotobosque en hábitats perturbados de selva lluviosa tropical. Caldasia 19:507520.

National Geographic. 1999. Field guide to the birds of North America, $3^{\text {rd }}$ edition. National Geographic Society, Washington, D. C. 480 p.

Newmark, W. D. 1991. Tropical forest fragmentation and the local extinction of understory birds in the eastern Usambara Mountains, Tanzania. Conservation Biology 5: 67-78.

Ortiz-Pulido, R., H. Gómez de Silva G., F. GonzálezGarcía y A. Álvarez A. 1995. Avifauna del Centro de Investigaciones Costeras la Mancha, Veracruz, México. Acta Zoológica Mexicana (n. s.) 66:87-118.

Pennington, T. D. y J. Sarukhán. 1998. Árboles tropicales de México. Universidad Nacional Autónoma de México y Fondo de Cultura Económica, México, D. F. 521 p.

Recher, H. F. 1969. Bird species diversity and habitat diversity in Australia and North America. American Naturalist 103:75-80.

Remsen, J. V. Jr. 1994. Use and misuse of bird lists in community ecology and conservation. Auk 111:225227.

Rice, J., Anderson, B.W. y Ohmart, R. D. 1994. Comparison of the importance of different habitat attributes to avian community organization. Journal of Wildlife Management 48: 895-911.

Rodríquez-Estrella, R., León de la Luz, L., Breceda, A., Castellanos A., Cancino, J. y Llinas, J. 1996. Status, density and habitat relationships of the endemic terrestrial birds of Socorro Island, Revillagigedo Islands, Mexico. Biological Conservation 76: 195202.

Rotenberry J. T. 1985. The role of habitat in avian community composition: physiognomy or floristics. 
Oecologia 67:213-217.

Roth, R. R. 1976. Spatial heterogeneity and bird species diversity. Ecology 57:773-782.

SAS Institute. 1999. SAS/STAT User's guide: statistics, statistical version 6. Cary, North Carolina.

Schulte LA, Niemi GJ. 1998. Bird communities of early successional burned and logged forest. Journal of Wildlife Management 62:1418-1429.

Sherry, T. W. y R. T. Holmes. 1995. Summer versus winter limitation of populations: what are the issues and what is the evidence? In Ecology and management of Neotropical migratory birds: a synthesis and review of critical issues, T. E. Martin y D. M. Finch (eds.). Oxford University Press, New York, p. 85-120.

Smith, A. L., J. Salgado O. y R. J. Robertson. 2001. Distribution patterns of migrant and resident birds in successional forests of the Yucatan Peninsula, Mexico. Biotropica 33: 153-170.

Staicer, C. A. 1992. Social behavior of the northern parula, cape may warbler, and prairie warbler wintering in second-growth forests in southwestern Puerto Rico.
In Ecology and conservation of neotropical migrant landbirds, J. M. Hagan y D. W. Johnston (eds.). Smithsonian Institution Press, Washington, DC., p. 308-320.

Stiles, F. G. y C. I. Bohórquez. 2000. Evaluando el estado de la Biodiversidad: el caso de la avifauna de la Serranía de las Quinchas, Boyacá, Colombia. Caldasia 22:61-69.

Snow, B. K. y D. W. Snow. 1971. The feeding ecology of tanagers and honeycreeper in Trinidad. Auk 88:291322.

Willson, M. F. 1974. Avian community organization and habitat structure. Ecology 55:1107-1029.

Wiens, J. A. 1997. Metapopulation dynamics and landscape ecology. In Metapopulation biology, I. A.Hanski y M. E. Gilpin (eds.). Academic, New York, p. 43-62.

Whitman, A. A., J. M. Hagan III, y N. V. L. Brokaw. 1997. A comparison of two bird survey techniques used in a subtropical forest. Condor 99:955-965.

Zar, J. H. 1996. Biostatistical Analysis. Prentice Hall, New Jersey. 662 p.

Apéndice 1. Avifauna registrada en las áreas y sitios de muestreo en Santa Gertrudis. La nomenclatura es la empleada por la Unión de Ornitólogos Americanos (AOU 2002). Estacionalidad. R: Residente, M: migratoria. Áreas de registro. 1= potrero, $2=$ selva con regeneración intermedia y $3=$ selva madura; cuando hay 2 o más áreas, la primera indica que la especie se registró ahí con mayor frecuencia. Los números de los sitios de muestreo indican el lugar donde se registró la avifauna dentro de cada área (e.g. Ortalis vetula se registró en las áreas 2 y 1 en el sitio 1 de cada una de estas). Abundancia, A: abundante, E: escasa y R: rara.

\begin{tabular}{lcccccc}
\hline Orden, familia y especie & Estacionalidad & Área de & & Sitios de muestro & Abundancia \\
registro & 1 & 2 & 3 & 4 & \\
\hline
\end{tabular}

\section{TINAMIFORMES}

\section{Tinamidae}

Crypturellus

cinnamomeus

GALLIFORMES

Cracidae

Ortalis vetula

Crax rubra

$\mathrm{R}$

$\mathrm{R}$

2, 1

1,2

3

2

A

CICONIFORMES

Cathartidae

Coragyps atratus

Cathartes aura

$\mathrm{R}$

FALCONIFORMES

Accipitridae

Accipiter striatus

M
2,1

1,2 
Buteo magnirostris

$\mathrm{R}$

Falconidae

Micrastur semitorquatus

Herpetotheres cachinnans

COLUMBIFORMES

Columbidae

Patagioenas flavirostris

Patagioenas nigrirostris

Columbina passerina

Columbina minuta

Leptotila verreauxi

Geotrygon montana

PSITTACIFORMES

\section{Psittacidae}

Amazona autumnalis

CUCULIFORMES

\section{Cuculidae}

Coccyzus americanus

Coccyzus minor

Piaya cayana

Crotophaga sulcirostris

STRIGIFORMES

\section{Strigidae}

Ciccaba virgata

CAPRIMULGIFORMES

Caprimulgidae

Nyctidromus albicollis

\section{APODIFORMES}

Trochilidae

Campylopterus curvipennis

Amazilia candida

Amazilia yucatanensis

Archilochus colubris

TROGONIFORMES

Trogonidae

Trogon melanocephalus

Trogon violaceus

Trogon collaris

Trogon massena

CORACIIFORMES

Momotidae

Momotus momota

Ramphastidae

Pteroglossus torquatus

Ramphastos sulfuratus
$\mathrm{R}$

$\mathrm{R}$

$\mathrm{R}$

$\mathrm{R}$

$\mathrm{R}$

$\mathrm{R}$

$\mathrm{R}$

$\mathrm{R}$

$\mathrm{R}$

M

$\mathrm{M}$

$\mathrm{R}$

$\mathrm{R}$

$\mathrm{R}$

$\mathrm{R}$

$\mathrm{R}$

$\mathrm{R}$

$\mathrm{R}$

$\mathrm{R}$

$\mathrm{R}$

$\mathrm{R}$

$\mathrm{R}$

$\mathrm{R}$

$\mathrm{R}$

R

$\mathrm{R}$
1

1

A

R

E

22

2

\section{E}

A

$\mathrm{R}$

$\mathrm{R}$

E

E

$\begin{array}{cc}2,3 & 2 \\ 2 & 2\end{array}$

$2,1 \quad 2$

$1,2 \quad 2$

A

$\mathrm{R}$

$\mathrm{R}$

$2,1,3 \quad 2,3 \quad 1,2,3 \quad 2,3 \quad 2,3$

A

A

A

A

A

E

A

E

$2,1 \quad 2 \quad 1$

E

A

E

$\mathrm{R}$

2 2 R

$\begin{array}{llllll}2,3 & 2 & 2,3 & 2 & 2 & \text { A }\end{array}$

$\begin{array}{cccccc}1,3 & 3 & & 1 & & \text { A } \\ 2,3 & 2,3 & 2 & 2 & 2 & \text { A }\end{array}$

A 
Picidae

Melanerpes aurifrons

Sphyrapicus varius

Piculus aeruginosus

Dryocopus lineatus

PASSERIFORMES

Dendrocolaptidae

Sittasomus griseicapillus

Xiphorhynchus flavigaster

Tyrannidae

Rhynchocyclus brevirostris

Empidonax occidentalis

Myiarchus tuberculifer

Myiarchus crinitus

Myiarchus tyrannulus

Pitangus sulphuratus

Megarynchus pitangua

Myiozetetes similis

Myiodynastes luteiventris

Tyrannus tyrannus

Tityra semifasciata

\section{Vireonidae}

Vireo griseus

Vireo flavifrons

Vireo cassinii

Vireo solitarius

Vireo huttoni

Vireo gilvus

Vireo olivaceus

Corvidae

Cyanocorax morio

\section{Paridae}

Baeolophus bicolor

Troglodytidae

Campylorhynchus rufinucha

Thryothorus maculipectus

Troglodytes aedon

Uropsila leucogastra

Henicorhina leucosticta

Sylviidae

Polioptila caerulea

Turdidae

Myadestes occidentalis

Catharus mexicanus

Catharus minimus
$\mathrm{R}$
$\mathrm{M}$
$\mathrm{R}$
$\mathrm{R}$

$2,1,3$

2, 3

$1,3 \quad 1,2,3$

A

$\mathrm{R}$

$\mathrm{R}$

A

$\begin{array}{llll}2,1 & 2 & 1 & 2\end{array}$

$\mathrm{E}$

A

$\mathrm{R}$

A

A

E

A

A

A

A

E

R

A

$\begin{array}{lllll}2,3,1 & 2 & 2 & 2 & 1,2,3\end{array}$

1,2

A

$\mathrm{R}$

A

A

$\mathrm{R}$

E

A

$\mathrm{R}$

$2,1,3$

$1,2,3 \quad 1,2,3 \quad 1,2,3$

A

E

$\mathrm{R}$

1

1

1

A

$\mathrm{R}$
$\mathrm{R}$

$\begin{array}{llll}1 & 1 & 1 & 1\end{array}$

$2,1 \quad 1,2 \quad 1,2 \quad 2 \quad 1,2$

A

$\mathrm{R}$

M

$\mathrm{R}$

R

$1,2,3$

1,2

$1,2 \quad 1$

1,3

A

$2,3,1 \quad 1,2,3 \quad 1,2,3 \quad 1,2,3 \quad 1,2,3$

A

M

2,1,

$1,2,3 \quad 1,2,3 \quad 2$

1,2

A

R

$\mathrm{R}$

M

\section{2}

2

2

2

R

$\mathrm{R}$

E 
Catharus ustulatus
Hylocichla mustelina
Turdus grayi
Turdus assimilis

Mimidae

Dumetella carolinensis

Parulidae

Vermivora pinus

Vermivora ruficapilla

Parula americana

Parula pitiayumi

Dendroica pensylvanica

Dendroica magnolia

Dendroica coronata

Dendroica virens

Mniotilta varia

Setophaga ruticilla

Helmitheros vermivorum

Limnothlypis swainsonii

Seiurus aurocapilla

Seiurus motacilla

Oporornis formosus

Geothlypis poliocephala

Wilsonia citrina

Wilsonia pusilla

Wilsonia canadensis

Euthlypis lachrymosa

Basileuterus culicivorus

Icteria virens

Thraupidae

Habia rubica

Habia fuscicauda

Cyanerpes cyaneus

\section{Emberizidae}

Sporophila torqueola

Tiaris olivaceus

Arremonops rufivirgatus

Aimophila rufescens

Cardinalidae

Saltator atriceps

Cardinalis cardinalis

Cyanocompsa parellina

Passerina cyanea

Passerina versicolor

Passerina ciris
M

M

$\mathrm{R}$

$\mathrm{R}$

M

M

$\mathrm{M}$

M

M

M

M

M

$\mathrm{M}$

M

$\mathrm{M}$

$\mathrm{M}$

M

$\mathrm{M}$

M

$\mathrm{M}$

$\mathrm{R}$

$\mathrm{M}$

$\mathrm{M}$

$\mathrm{M}$

$\mathrm{R}$

$\mathrm{R}$

$\mathrm{M}$

$\mathrm{R}$

$\mathrm{R}$

R

$\mathrm{R}$

$\mathrm{R}$

$\mathrm{R}$

$\mathrm{R}$

R

$\mathrm{R}$

R

M

R

M

$2,1,3$

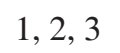

2

2

2,3

2

$1,2,3$

$1,3 \quad 1,3$

1,2

A

$1,2 \quad 1,2 \quad \mathrm{E}$

$\begin{array}{lllll}2,1 & 2 & 1 & 2 & \mathrm{E}\end{array}$

$\begin{array}{llllll}2,3 & 2,3 & 2 & 2,3 & 2 & \text { A }\end{array}$

$2,1,3 \quad 1,2,3 \quad 1,2,3 \quad 1,2,3 \quad 1,2,3 \quad$ A

1 R R

$\begin{array}{llll}2,1 & 1,2 & 1,2 & \mathrm{~A}\end{array}$

1 1 $\quad$ R

$\begin{array}{llllll}2,1,3 & 1,2 & 1,2 & 1,2,3 & 1,2 & \mathrm{~A}\end{array}$

$\begin{array}{llllll}2,1,3 & 1,2,3 & 2,3 & 2,3 & 1,2 & \mathrm{~A}\end{array}$

$\begin{array}{llll}1 & 1 & 1 & \mathrm{E}\end{array}$

$\begin{array}{lllll}2 & 2 & 2 & 2\end{array}$

222 E

$2,1,3 \quad 1,2,3 \quad 1,2,3 \quad 1,2 \quad 1,2,3 \quad$ A

$3 \quad 3 \quad$ A

$2 \quad 2 \quad 2 \quad$ A

1 1 1

$\begin{array}{llllll}2,1 & 1,2 & 2 & 2 & 1,2 & \text { A }\end{array}$

$2,1,3 \quad 1,2,3 \quad 1,2 \quad 1,2,3 \quad 1,2,3 \quad$ A

$\begin{array}{llll}2,1 & 1,2 & 2 & \mathrm{E}\end{array}$

$\begin{array}{llllll}2,3,1 & 2,3 & 2,3 & 2,3 & 1,2,3 & \text { A }\end{array}$

$2,3,1 \quad 1,2,3 \quad 1,2,3 \quad 1,2,3 \quad 2,3 \quad$ A

$\begin{array}{llllll}2,1 & 1,2 & 1 & 1 & 1 & \text { A }\end{array}$

$\begin{array}{cccccc}2 & 2 & 2 & 2 & & \mathrm{~A} \\ 2,3 & 2,3 & 2,3 & 2,3 & 2,3 & \mathrm{~A} \\ 2,1 & & 2 & & 1 & \mathrm{~A}\end{array}$

A

$\mathrm{R}$

A

A

$\mathrm{R}$

1 1

$\begin{array}{ccccccc}\mathrm{R} & 2 & & & & 2 & \mathrm{E} \\ \mathrm{R} & 1 & 1 & 1 & 1 & 1 & \mathrm{~A} \\ \mathrm{M} & 2,1,3 & 1,2,3 & 1,2,3 & 1,2,3 & 1,2 & \mathrm{~A} \\ \mathrm{R} & 2 & & 2 & & 2 & \mathrm{E} \\ \mathrm{M} & 1 & 1 & & 1 & 1 & \mathrm{E} \\ \mathrm{R} & 2,1 & 2 & 2 & 1 & 1 & \mathrm{E}\end{array}$




\section{Icteridae}

Dives dives

Molothrus aeneus

Icterus gularis

Psarocolius montezuma

Fringillidae

Euphonia affinis

Euphonia hirundinacea

$\begin{array}{lcccccc}\mathrm{R} & 2 & 2 & & & & \mathrm{~A} \\ \mathrm{R} & 1 & & & & 1 & \mathrm{~A} \\ \mathrm{R} & 2 & 2 & & & & \mathrm{R} \\ \mathrm{R} & 2,3,1 & 1,2,3 & 2,3 & 2,3 & 2,3 & \mathrm{~A} \\ \mathrm{R} & 2,1,3 & 2,3 & 1,2,3 & 1,2 & 1,2 & \mathrm{~A} \\ \mathrm{R} & 2,3 & 2,3 & 2,3 & 2,3 & 2,3 & \mathrm{~A}\end{array}$

A

A

A

A

A 